удК 624.131 .3

\title{
ЧИСЛЕННОЕ ИССЛЕДОВАНИЕ УСТОЙЧИВОСТИ ГРУНТА С ИСПОЛЬЗОВАНИЕМ ГЕОФИЗИЧЕСКИХ ДАННЫХ
}

\author{
Татаурова Антонина Андреевна ${ }^{1}$, \\ TataurovaAA@ipgg.sbras.ru \\ Мельник Елена Александровна ${ }^{1}$, \\ MelnikEA@ipgg.sbras.ru \\ Стефанов Юрий Павлович1, \\ yu_st@mail.ru \\ 1 Институт нефтегазовой геологии и геофизики им. А.А. Трофимука СО РАН, \\ Россия, 630090, г. Новосибирск, пр. Коптюга, 3.
}

\begin{abstract}
Актуальность работы. Строительство промышленных объектов на участках со сложным рельефом и геологическим строением нередко требует проведения расчетов напряженно-деформированного состояния с целью получения оценок возможных зон развития необратимой деформации. Данная задача может быть решена с помощью численного моделирования. В настоящей работе рассмотрен участок планируемого строительства электродепо «Волочаевское» Дзержинской линии Новосибирского метрополитена. Сложный рельеф и низкие прочностные характеристики зоны строительства требуют особого внимания к оценке несущей способности грунта и устойчивости склонов близкорасположенных оврагов. Применение численного моделирования для оценки напряженно-деформированного состояния среды с использованием геофризических данных о ее строении и механических свойствах позволили получить информацию о зонах концентрации напряжений и возможного развития необратимой деформации.

Цель: определить существующее напряженно-деформированное состояние и оценить устойчивость верхней части геологического разреза на основе имеющихся петрофизических и геофизических данных, полученных с использованием сейсмо- $и$ электроразведки.

Объект: геологический разрез, проходящий вдоль участка продления Дзержинской линии Новосибирского метрополитена электродепо «Волочаевское».

Методы. Расчет напряженно-деформированного состояния грунта в зоне строительства осуществлялся при помощи численного моделирования. Задача решалась в упругопластической постановке. Геометрические и геомеханические параметры среды были получены в ходе анализа петрофизических и геофизических данных.

Результаты. Рассмотрено сечение среды, которое пересекает участок строительства электродепо «Волочаевское» Дзержинской линии Новосибирского метрополитена. Представлены две геомеханические модели. Первая горизонтальнослоистая и построена на основе сейсморазведочных данных. Вторая более сложная, учитывает также данные о неоднородностях, выделенных на основе распределения удельного электрического сопротивления. Показано, что для исследуемой территории характерна общая деформация, связанная с наличием зон, обладающих пониженной прочностью, которые выделяются при комплексном анализе данных электроразведки и петрофизики. Несмотря на то, что учет этих зон не привел к значимым изменениям в напряженно-деформированном состоянии среды, было показано, что в приповерхностной части земной коры возможно формирование зон локализации необратимой деформации и трещиноватости. Установлено, что зоны $\mathrm{C}$ резким перепадом рельефра представляют наибольшую опасность и будут разрушаться в первую очередь.
\end{abstract}

\section{Ключевые слова:}

Геомеханика, численное моделирование, напряженно-деформированное состояние, деформация, разрушение, геофизические исследования, г. Новосибирск.

\section{Введение}

Оценка возможного развития деформационных процессов и предотвращения достижения критических величин в грунтах является важной геологической задачей $[1,2]$. С этой целью осуществляется расчленение геологического разреза и установление литологических границ, выявление и прослеживание неоднородностей строения массива грунтов, а также тектонических нарушений, зон повышенной трещиноватости и обводненности [3]. Эти данные позволяют провести оценку напряженно-деформированного состояния среды. Решение подобных задач является важным для инженерной геологии, и для этого все чаще прибегают к использованию геомеханического моделирования [4-6], в частности к использованию упругопластической модели [7-9]. Такой подход поз- воляет выделить зоны локализации пластических деформаций (зоны разрушений). Основная цель при этом не только построить модель, отвечающую существующему напряженному состоянию, но также осуществить контроль за состоянием среды.

Эти задачи особенно актуальны для областей со склонами, так как они могут создавать проблемы при градостроительном освоении этих территорий [10]. Помимо этого, большое значение имеют вопросы, связанные с наличием неоднородностей в среде выраженных разным составом грунтов и влиянием обводненных зон [11]. Все эти аспекты требуют учета в процессе построения модели и расчета ее деформирования.

Участок продления Дзержинской линии Новосибирского метрополитена электродепо «Волочаевское» 
имеет два склона различной крутизны, поэтому необходимо контролировать их динамическое состояние и дать прогнозную оценку развития деформации грунта.

\section{Методика работы}

Одной из основных задач геомеханических исследований является получение оценок напряженнодеформированного состояния геологической среды и прогноз ее поведения в заданных условиях. В данной работе основное внимание уделено выявлению зон пластической деформации, которые представляют наибольшую опасность из-за развития в них необратимой деформации при изменении нагрузки, вызванной строительством и эксплуатацией сооружений.

Решение поставленной задачи осуществлялось при помощи численного моделирования, с использованием апробированного пакета компьютерных программ для решения двумерных геомеханических задач [9, 12-14].

Поведение среды описывалось в рамках модели Друккера-Прагера с неассоциированным законом течения $[15,16]$. Согласно принятой модели, будем считать, что скорость деформации представляет сумму упругой и пластической (неупругой) частей. Соответственно, напряженное состояние среды рассчитывается из уравнения:

$$
\dot{\sigma}_{i j}=\lambda\left(\theta-\theta^{p}\right) \delta_{i j}+2 \mu\left(d_{i j}-d_{i j}^{p}\right),
$$

где $\lambda$ и $\mu$ - коэффициенты Ламе; $\delta_{i j}-$ символ Кронекера. Компоненты тензоров скоростей деформаций $d_{i j}$ определяются соотношениями Коши:

$$
d_{i j}=\frac{1}{2}\left(u_{i, j}+u_{j, i}\right), \theta=d_{k k} .
$$

Упругое состояние среды в пространстве напряжений ограничено поверхностью предельного состояния:

$$
f=\tau-\alpha p-Y
$$

где

$$
\tau=\sqrt{\left(\left(\sigma_{1}-\sigma_{2}\right)^{2}+\left(\sigma_{2}-\sigma_{3}\right)^{2}+\left(\sigma_{3}-\sigma_{1}\right)^{2}\right) / 6}
$$

- интенсивность касательных напряжений; $p=-\left(\sigma_{1}+\sigma_{2}+\sigma_{3}\right) / 3$ - давление; $\alpha$ и $Y$ - параметры, которые могут быть выражены через коэффициенты внутреннего трения $\varphi$ и сцепления $C$ модели КулонаМора с помощью соотношений [15]:

$$
\alpha=\frac{2 \sin \varphi}{\sqrt{3}(3+\sin \varphi)} ; Y=\frac{6 C \cos \varphi}{\sqrt{3}(3+\sin \varphi)} .
$$

Рассматривалась задача о напряженно-деформированном состоянии горизонтально-слоистой среды со сложным рельефом под действием массовых гравитационных сил. Предполагалось, что слои лежат на жестком основании, на боковых границах отсутствуют горизонтальные смещения: $\mathrm{u}_{\mathrm{x}}=0$.

Задача решалась в двумерной постановке для условий плоской деформации. Расчеты осуществлялись при помощи явной конечно-разностной схемы [17].

Для анализа напряженного состояния и оценки того, насколько состояние среды близко к критическому в момент, когда начинает развиваться необратимая деформация и разрушение, в геомеханике широко используются кулоновские напряжения [18-20]; $\tau_{\mathrm{C}}=T-\operatorname{tg}(\varphi) \sigma$. Как видно из соотношения, данный параметр представляет собой разность между касательными напряжениями на площадке и предельным трением. Соответственно, разрушение происходит при достижении параметром величины сцепления, а положительная величина данного параметра говорит о том, что касательные напряжения преодолевают трение в случае разрушения.

По аналогии введем параметр нормированных кулоновских напряжений, но для модели ДруккераПрагера:

$$
F=(\tau-\alpha p) / Y \text {. }
$$

Достижение данным параметром значения $F=1$ соответствует тому, что напряжения достигли предела упругости и в данной области возможно развитие пластической деформации и разрушение среды. Положительное значение данного параметра $0<F<1$ означает, что сдвиговые напряжения превышают трение между частицами среды, но сцепление между ними не допускает развитие пластической деформации и среда остается в упругом состоянии. Однако, учитывая низкое сцепление грунта, такое состояние может быть потенциально опасным.

\section{Геомеханическое моделирование}

В работе проведена оценка напряженнодеформированного состояния расчетной области. Геометрия среды и ее механические свойства определялись на основе геофизических и петрофизических данных.

Предложена геомеханическая модель для геологического разреза, проходящего через район электродепо «Волочаевское». Его протяженность составила 1100 м и мощность 105 м (рис. 1). Все геомеханические параметры среды представлены в таблице. Они были получены на основе анализа данных бурения и петрофизических исследований.

Рассматриваемый район сложен осадочными породами, которые представлены различными фракциями. На основе данных о скоростях пробега продольных и поперечных волн и лабораторных измерений на керне, полученных в процессе бурения [21], было выделено три слоя с различными механическими свойствами (рис. $1, a$ ). Первый слой представлен супесями с линзами и маломощными прослоями суглинков (таблица). Второй слой представлен алевролитами различных фракций, и третий - супесями пылевыми и песчаными с прослоями песка. Информация о четвертом слое отсутствует, он был введен с предположением о том, что он сложен песчаником. Данная модель позволила определить влияние рельефа и геометрии границ слоев на распределение напряженно-деформированного состояния, а также получить оценки развития необратимой деформации.

По данным электроразведки были выделены четыре зоны (геологические тела № 5-8, таблица) с различной величиной удельного электрического сопротивления, что объясняется составом пород и степенью обводне- 
ности. По данным электроразведки и бурения участки 6 и 8 являются наиболее водонасыщенными начиная с глубины 5-7 м. В данных зонах было задано пониже- ние значения коэффициента внутреннего трения до величин порядка 0,15-0,18 [21]. Модель среды с учетом этих зон представлена на рис. 1, б.

a

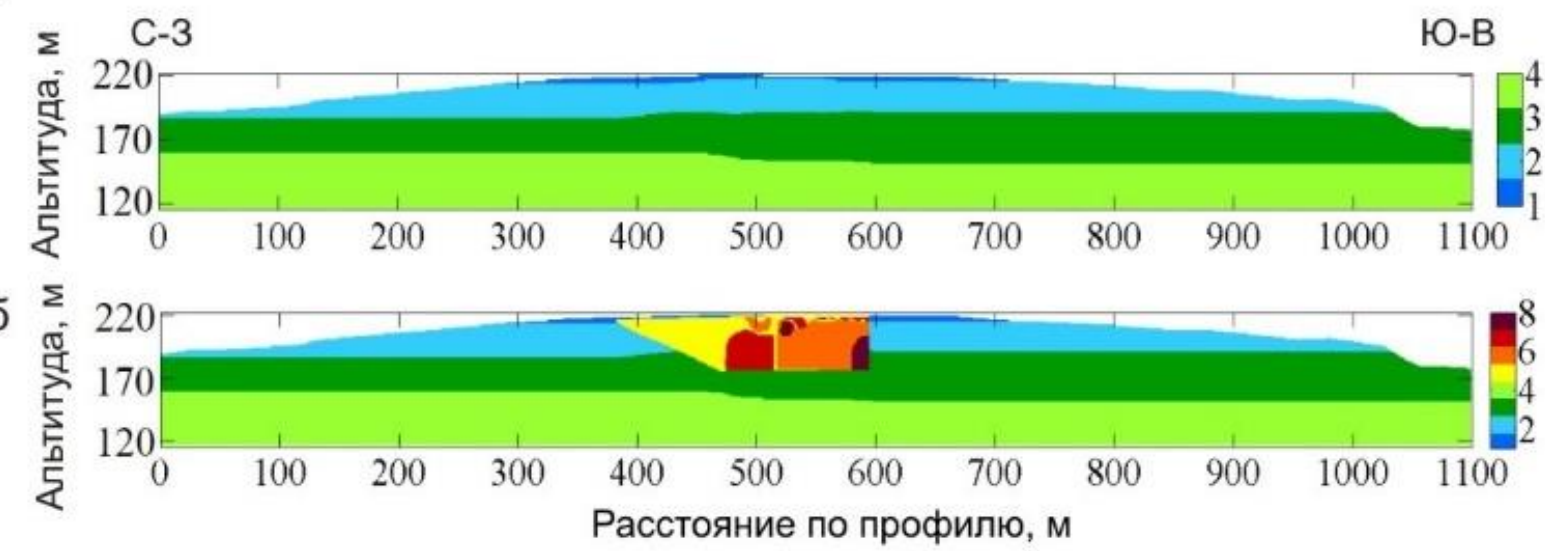

Pис. 1. Модели среды: а) по сейсмоплотностным данным; б) с учетом зон, выделенных на основе электропроводности среды и анализа керна. Показана иветовая шкала с нумерачией слоев, свойства которых приведены в таблице

Fig. 1. Environmental models: a) according to seismic density data; б) taking into account the zones identified on the basis of the electrical conductivity of the medium and core analysis. A color scale is shown with numbering of layers which properties are shown in Table

Таблица. Геомеханические параметры слоев

Table. Geomechanical parameters of layers

\begin{tabular}{|c|c|c|c|c|c|c|}
\hline $\begin{array}{c}\text { Геологические } \\
\text { тела } \\
\text { Geological body }\end{array}$ & $\begin{array}{c}\text { Плотность, } \rho \\
\left(\text { кг } / \mathrm{M}^{3}\right) \\
\text { Density } \\
\left(\mathrm{kg} / \mathrm{m}^{3}\right)\end{array}$ & $\begin{array}{l}\mu \text { коэф. } \\
\text { Пуассона } \\
\text { Poisson's } \\
\text { ratio }\end{array}$ & \begin{tabular}{|c|} 
Модуль упру- \\
гости, $E$ \\
(МПа) \\
Elastic modulus \\
(MPa) \\
\end{tabular} & $\begin{array}{c}\text { Параметр } \\
\text { ды, } Y \text { (MПа) } \\
\text { Model } \\
\text { ter, } Y \\
\text { (MPa) }\end{array}$ & $\begin{array}{c}\text { Параметр } \\
\text { среды, } \alpha \\
\text { Model } \\
\text { parameter, } \\
\alpha\end{array}$ & $\begin{array}{c}\text { Состав } \\
\text { Composition }\end{array}$ \\
\hline 1 & 1,79 & 0,31 & 207 & 0,018 & 0,21 & $\begin{array}{l}\text { Супеси с линзами и маломощными } \\
\text { прослоями суглинков } \\
\text { Sandy loam with lenses and thin layers } \\
\text { of loam }\end{array}$ \\
\hline 2 & 1,98 & 0,28 & 292 & 0,027 & 0,2 & $\begin{array}{l}\text { Алевролиты разной фракции } \\
\text { Aleurolites of different fractions }\end{array}$ \\
\hline 3 & 1,82 & 0,29 & 294 & 0,019 & 0,21 & $\begin{array}{l}\text { Супеси пылевые и песчаные с про- } \\
\text { слоями песка } \\
\text { Sandy loam with sand }\end{array}$ \\
\hline 4 & 1,99 & 0,27 & 380 & 0,036 & 0,3 & $\begin{array}{l}\text { Песчаники } \\
\text { Sandstone }\end{array}$ \\
\hline 5 & 1,98 & 0,28 & 292 & 0,024 & 0,18 & $\begin{array}{l}\text { Супеси с линзами и маломощными } \\
\text { прослоями суглинков } \\
\text { Sandy loam with lenses and thin layers } \\
\text { of loam }\end{array}$ \\
\hline 6 & 1,98 & 0,28 & 292 & 0,018 & 0,16 & $\begin{array}{l}\text { Пластичные супеси } \\
\text { Plastic sandy loam }\end{array}$ \\
\hline 7 & 1,98 & 0,28 & 292 & 0,017 & 0,17 & $\begin{array}{l}\text { Крупнозернистые алевролитовые } \\
\text { разности } \\
\text { Aleurolites of large fractions }\end{array}$ \\
\hline 8 & 1,98 & 0,28 & 292 & 0,015 & 0,15 & $\begin{array}{l}\text { Пластичные супеси } \\
\text { Plastic sandy loam }\end{array}$ \\
\hline
\end{tabular}

В результате расчетов получены оценки устойчивости напряженно-деформированного состояния исследуемой модели среды. Для этого определены зоны, в которых возникает пластическая деформация. Кроме того, было рассчитано распределение нормированных кулоновских напряжений, которые отражают, насколько состояние среды близко к пластическому.

Результаты расчетов для модели, построенной только по сейсмоплотностным данным, показывают, что значения параметра состояния $F$, близкое к 1 , наблюдаются в двух областях (рис. 2). Первая из них расположена в центральной части профиля и прослеживается на всю глубину модели, что обусловлено наибольшей мощностью, а значит, и самыми высокими значениями напряжений, вызванных гравитационными силами. Вторая область с критическим значением параметра $F$ располагается в зоне резкого изменения рельефа в северо-восточной части профиля, где 
имеется наиболее крутой склон оврага. Резкие перепады напряжений в этой области приводят к неустойчивости рассматриваемого участка. Несмотря на высокие значения нормированных кулоновских напряжений, величина интенсивности сдвиговой пластической деформации остается малой и не превышает $0,02 \%$. В связи с этим можно предположить, что для данного участка может возникнуть небольшая усадка среды в центральной части разреза.

Второй вариант расчета напряженно-деформированного состояния с учетом аномалий, полученных методом электроразведки, показывает, что наличие участков обводнения (участоки с пониженной прочностью) для модели не привело к существенным из- менениям напряженно-деформированного состояния (рис. 3). Область, в которой нормированные кулоновские напряжения принимают значение $F=1$, практически не изменилась по сравнению с результатом для слоистой модели (рис. $3, a$ ). В то же время величина пластических деформаций возросла незначительно. В центральной части область пластической деформации, превышающая $0,02 \%$, расширилась (рис. 3,6 ), что может привести к большей усадке среды, чем в первом случае. Неизменной остается величина интенсивности сдвиговой пластической деформации на северо-восточной части профиля (борт склона).

a

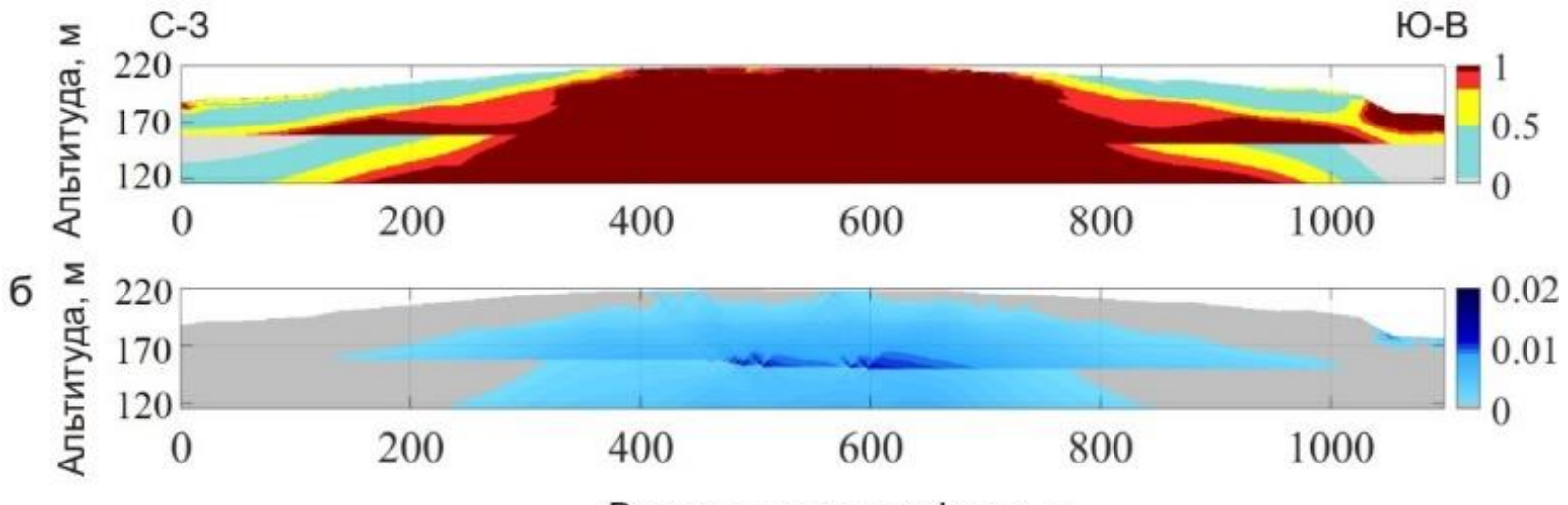

Расстояние по профилю, м

Рис. 2. Нормированные кулоновские напряжения (а) и интенсивность сдвиговых пластических деформаций (\%) (б) Fig. 2. Normalized Coulomb stresses (a) and intensity of shear plastic deformations (\%) (б)

a

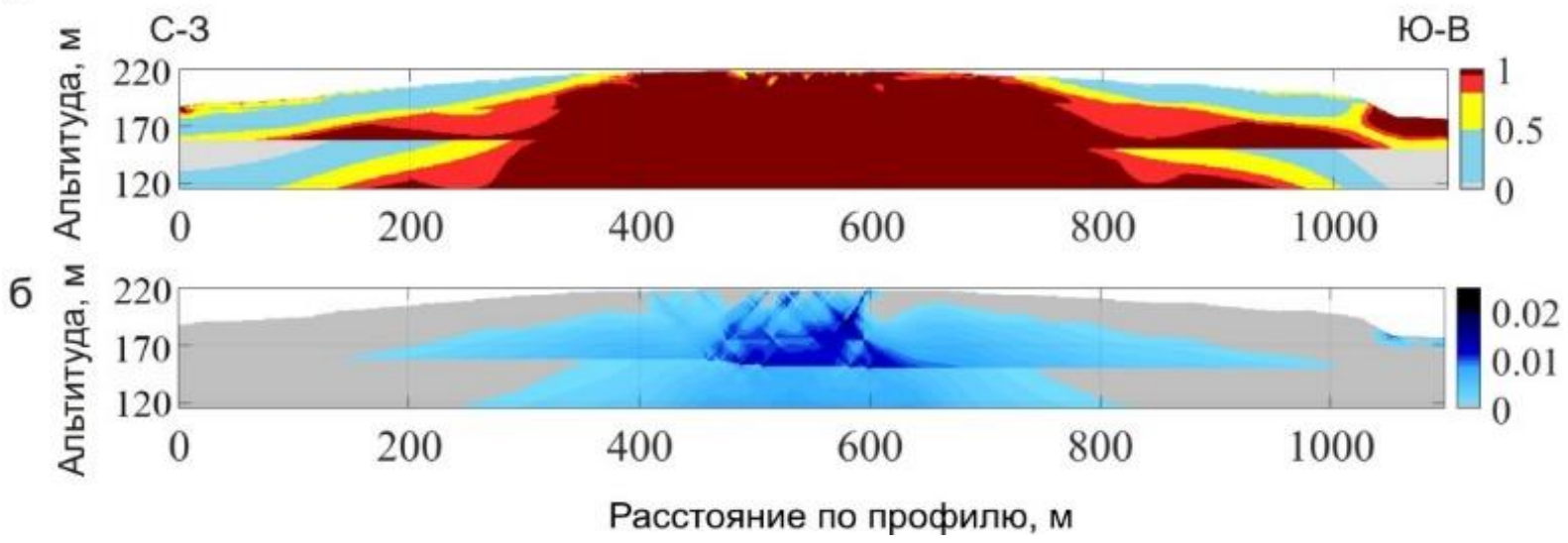

Рис. 3. Нормированные кулоновские напряжения (а) и интенсивность сдвиговых пластических деформаций (\%) (б) с учетом аномалий, полученных методом электроразведки

Fig. 3. Normalized Coulomb stresses (a) and intensity of shear plastic deformations (\%) (б) taking into account anomalies obtained by the method of electrical exploration

\section{Заключение}

Таким образом, в результате анализа напряженнодеформированного состояния среды было установлено, что наибольшую опасность могут представлять зоны резкого изменения рельефа (в нашем случае при угле $30^{\circ}$ ), что приводит к неоднородному распределению напряжений. Это может служить причиной обрушений и неустойчивости среды. В связи с не- большими изменениями рельефа поверхности при деформации среды под собственным весом можно говорить об устойчивости района размещения электродепо «Волочаевское» и рекомендовать укрепить области с резкими перепадами рельефа для предупреждения возможных разрушений. Учет неоднородностей с пониженной прочностью не привел к значительным изменениям напряженно-деформированного 
состояния в среде, хотя интенсивность пластической деформации достигла несколько большего значения. Это может привести к большей усадке среды, чем в случае рассмотренной нами слоистой среды.

Исследование выполнено при поддержке проекта ФНИ № 0331-2019-0006 «Структуры и напряженно-

\section{СПИСОК ЛИТЕРАТУРЫ}

1. Анализ причин аварий зданий и сооружений и пути повышения их надежности / В.В. Леденев., В.Г. Однолько // Вестник ТГТУ. Машиностроение. Металлообработка. Строительство. 2012. - T. 18. - № 2. - C. 449-457.

2. Итоги изучения напряженно-деформированного состояния массивов горных пород / Э.В. Калинин // Инженерная геология. - 2017. - № 6. - С. 58-64.

3. Осипов В.И. Крупномасштабное геологическое картирование территории г. Москвы // Геоэкология. Инженерная геология, гидрогеология, геокриология. - 2011. - № 3. - С. 195-197.

4. Фоменко И.К. Математическое моделирование напряженного состояния инженерно-геологического массива, сложенного анизотропными горными породами: дис. ... канд. геол.минерал. наук. - М., 2000. - 92 с.

5. Фоменко И.К. Современные тенденции в расчетах устойчивости склонов // Инженерная геология. - 2012. - № 6. - С. 44-53.

6. Wang X., Wang H., Liang R.Y. A method for slope stability analysis considering subsurface stratigraphic uncertainty // Landslides. - 2018. - V. 15. - № 5. - P. 925-936.

7. Elasto-plastic modeling of soft soil considering degradation of stiffness / H. Zhang, Q. Chen, J. Chen, J. Wang // Journal of Shanghai Jiaotong University. - 2015. - V. 20. - № 6. - P. 683-689.

8. A review of basic soil constitutive model for geotechnical application / S.T. Kok, B.H.H. Bujang, N. Jamaloddin, S.J. Moh'd, S.S. Gue // Electronic Journal of Geotechnical Engineering. 2009. - V. 14J. - P. 1-18.

9. Геомеханические условия роста Тянь-Шаня и Алтая В.Д. Суворов, Ю.П. Стефанов, Е.В. Павлов, Е.А. Мельник, А.А. Татаурова, В.А. Кочнев // Доклады РАН. - 2017. T. 476. - № 5. - C. 562-566.

10. Zhu D., Griffiths D.V., Fentons G.A. Probabilistic stability analyses of layered excavated slopes // Geotechnique Letters. 2019. - V. 9. - № 3. - P. 1-4.

11. Chen Y., Marinelli F., Buscamera G. Mathematical interpretation of delayed instability in viscous unsaturated soil // Geotechnique Letters. - 2019. - V. 9. - № 3. - P. 1-8. деформированное состояние земной коры платформенных и складчатых областей Центральной Азии на основе совместного решения обратных задач сейсмологии и гравиметрии» и проекта РФФИ и Министерства образования, науки и инновационной политики Новосибирской области № 17-45-540528.

12. Стефанов Ю.П. Локализация деформаций и нарушений в геоматериалах. Численное моделирование // Физическая мезомеханика. - 2002. - Т. 5. - № 5. - С. 107-118.

13. Стефанов Ю.П. Некоторые особенности численного моделирования поведения упруго-хрупкопластичных материалов // Физическая мезомеханика. - 2005. - Т. 8. - № 3. - С. 129-142.

14. Стефанов Ю.П., Бакеев Р.А., Формирование цветковых структур нарушений в слое геосреды при разрывном горизонтальном сдвиге основания // Физика Земли. - 2015. - № 4. - С. 81-93.

15. Drucker D.C., Prager W. Soil mechanics and plastic analysis or limit design // Quarterly of Applied Mathematics. - 1952. V. 10. - № 2. - P. 157-165.

16. Nikolaevskii V.N. Governing equations of plastic deformation of a granular medium // J. Appl. Math. Mech. - 1971. - V. 35. - № 6. P. $1017-1029$.

17. Wilkins M.L. Computer simulation of dynamic phenomena. Berlin; Heidelberg; New York: Springer Verlag, 1999. - 246 p.

18. Ребецкий Ю.Л. Тектонические напряжения и области триггерного механизма возникновения землетрясений // Физическая мезомеханика. - 2007. - Т. 10. - № 1. - С. 25-37.

19. Cocco M., Rice J.R. Pore pressure and poroelasticity effects in Coulomb stress analysis of earthquake interactions // JGR Solid Earth. - 2002. - V. 107. - № B2. - P. ESE 2-1-ESE 2-17.

20. Evaluating models of Coulomb stress transfer: Is variable fault geometry important? / Z.K. Mildon, S. Toda, J. Walker, G. Roberts // Geophysical Research Letters. - 2016. - V. 43. № 24. - P. 12,407-12,414.

21. Изучение структурных и вещественных особенностей грунтов по комплексу сейсмоплотностных и геоэлектрических данных / Е.А. Мельник, А.А. Татаурова, В.В. Оленченко, В.Н. Глинских // Естественные и технические науки. - 2019. - № 2. C. $66-70$

Поступила 12.03.2020 2.

\section{Информация об авторах}

Tamaypoвa A.A., научный сотрудник Лаборатории глубинных геофизических исследований и региональной сейсмичности Института нефтегазовой геологии и геофизики им. А.А. Трофимука СО РАН.

Мельник E.A., кандидат геолого-минералогических наук, заведующая Лабораторией глубинных геофизических исследований и региональной сейсмичности Института нефтегазовой геологии и геофизики им. А.А. Трофимука СО РАН.

Стефанов Ю.П., доктор физико-математических наук, ведущий научный сотрудник Лаборатории глубинных геофизических исследований и региональной сейсмичности Института нефтегазовой геологии и геофизики им. А.А. Трофимука СО РАН. 
UDC 624.131 .3

\title{
EVALUATION OF SOIL STABILITY BY NUMERICAL MODELING
}

\author{
Antonina A. Tataurova ${ }^{1}$, \\ TataurovaAA@ipgg.sbras.ru
}

\author{
Elena A. Melnik' ${ }^{1}$, \\ MelnikEA@ipgg.sbras.ru \\ Yurii P. Stefanov 1 , \\ yu_st@mail.ru \\ 1 Institute of Petroleum Geology and Geophysics SB RAS, \\ 3, Koptyug avenue, Novosibirsk, 630090, Russia.
}

\begin{abstract}
The relevance of the research. The construction of industrial facilities in areas with difficult relief and geological structure requires calculations of the stress-strain state in order to obtain estimates of the zones of irreversible deformation development. This problem can be solved using numerical modeling. In this paper, we consider a section of the planned construction of the Volochaevskoe electric depot of the Dzerzhinsky line of the Novosibirsk subway. The difficult relief and low strength characteristics of the construction zone require special attention to assess of the soil bearing capacity and the slopes stability of closely located ravines. Numerical modeling is used to assess the stress-strain state of the medium using geophysical data on the structure and mechanical properties. These data provided information on stress concentration zones and the possible development of irreversible deformation.
\end{abstract}

The main aim of the research is to determine the stress-strain state and evaluate the stability of the upper part of the geological section using petrophysical and geophysical data obtained by seismic and electrical exploration.

The object: geological section along the extension section of the Dzerzhinsky line of the Novosibirsk subway of the Volochaevskoe electric depot.

Methods. Numerical modeling is used to calculate the stress-strain state in the construction zone. The problem is solved in an elastoplastic setting. Geometric and geomechanical parameters of the environment are obtained in the analysis of petrophysical and geophysical data.

Result. The paper considers the section of the medium crossing the construction site of the electric depot «Volochaevskoye» of the Dzerzhinsky line of the Novosibirsk subway. Two geomechanical models are introduced: the first horizontally layered built on the basis of seismic data, the second is more complex, it includes data on heterogeneities found on the basis of the electrical resistivity distribution. The study area is characterized by a general deformation associated with zones of reduced strength. They are found in a complex analysis of electrical exploration and petrophysics. The stress-strain state does not significantly change taking into account these zones. Localization zones of irreversible deformation and fracturing can be formed in the upper part of the earth's crust. It was established that zones with a sharp difference in relief represent the greatest danger and will be destroyed first.

\section{Key words:}

Geomechanics, numerical modeling, stress-strain state, deformation, fracture, geophysical research, Novosibirsk.

The research was supported by the FSR project no. 0331-2019-0006 «Structures and stress-strain state of the earth's crust of platforms and folded areas of Central Asia based on joint solution of inverse problems of seismology and gravimeter" and the RFBR project and the Ministry of Education, Science and Innovation policy of Novosibirsk region no. 17-45-540528.

\section{REFERENCES}

1. Ledenev V.V., Odnolko V.G. The analysis of causes of accidents of buildings and structures and ways to improve their reliability. Transactions TGTU, 2012, vol. 18, no. 2, pp. 449-457. In Rus.

2. Kalinin E.V. The results of the study stress-strain state of rock massifs. Engineering Geology World, 2017, no. 6, pp. 58-64. In Rus.

3. Osipov V.I. Krupnomasshtabnoe geologicheskoe kartirovanie territorii g. Moskvy [Large-scale geological mapping of the Moscow territory]. Environmental Geoscience, 2011, no. 3, pp. 195-197.

4. Fomenko I.K. Matematicheskoe modelirovanie napryazhennogo sostoyaniya inzhenerno-geologicheskogo massiva, slozhennogo anizotropnymi gornymi porodami. Diss. Kand. nauk. [Mathematical modeling of the stress state of an engineering-geological massif composed of anisotropic rocks. Cand. Diss.]. Moscow, 2000. $92 \mathrm{p}$.

5. Fomenko I.K. Current trends in slope stability calculations. Engineering Geology World, 2012, no.6, pp. 44-53. In Rus.

6. Wang X., Wang H., Liang R.Y. A method for slope stability analysis considering subsurface stratigraphic uncertainty. Landslides, 2018, vol. 15 , no. 5, pp. 925-936.
7. Zhang H., Chen Q., Chen J., Wang J. Elasto-plastic modeling of soft soil considering degradation of stiffness. Journal of Shanghai Jiaotong University, 2015, vol. 20, no. 6, pp. 683-689.

8. Kok S.T., Bujang B.H.H., Jamaloddin N., Moh'd S.J., Gue S.S. A review of basic soil constitutive model for geotechnical application. Electronic Journal of Geotechnical Engineering, 2009, vol. 14J, pp. 1-18.

9. Suvorov V.D., Stefanov Yu.P., Pavlov E.V., Melnik E.A., Tataurova A.A., Kochnev V.A. Geomekhanicheskiye usloviya rosta Tyan-Shanya i Altaya [Geomechnical conditions of the Tien Shan and Altai orogen]. Doklady RAN, 2017, vol. 476, no. 5, pp. 562-566.

10. Zhu D., Griffiths D.V., Fentons G.A. Probabilistic stability analyses of layered excavated slopes. Geotechnique Letters, 2019, vol. 9, no. 3, pp. 1-4.

11. Chen Y., Marinelli F., Buscamera G. Mathematical interpretation of delayed instability in viscous unsaturated soil. Geotechnique Letters, 2019, vol. 9, no. 3, pp. 1-8.

12. Stefanov Yu.P. Lokalizatsiya deformatsiy i narusheniy v geomaterialakh. Chislennoe modelirovanie [Deformation localization and fracture in geomaterials. Numerical simulation]. Fizicheskaya mezomekhanika, 2002, vol. 5, no. 5, pp. 107-118. 
13. Stefanov Yu.P. Nekotorye osobennosti chislennogo modelirovaniya povedeniya uprugo-khrupkoplastichnykh materialov [On some features of numerical simulation of the behavior of elastic-brittleplastic materials]. Fizicheskaya mezomekhanika, 2005, vol. 8, no. 3, pp. 129-142.

14. Stefanov Yu.P., Bakeev R.A. Formirovaniye tsvetkovykh struktur narusheniy v sloye geosredy pri razryvnom gorizontalnom sdvige osnovaniya [Formation of flower structures in a geologycal layer at a strike-slip displacement in the basement]. Fizika Zemli, 2015, no. 4, pp. 81-93.

15. Drucker D.C., Prager W. Soil mechanics and plastic analysis or limit design. Quarterly of Applied Mathematics, 1952, vol. 10, no. 2 , pp. $157-165$.

16. Nikolaevskii V.N. Governing equations of plastic deformation of a granular medium. J. Appl. Math. Mech., 1971, vol. 35, no. 6, pp. 1017-1029.
17. Wilkins M.L. Computer simulation of dynamic phenomena. Berlin, Heidelberg, New York, Springer Verlag Publ., 1999. 246 p.

18. Rebetsky Yu.L. Tectonic stresses and zones of earthquake triggering. Physical Mesomechanics, 2007, vol. 10, no. 1, pp. 25-37. In Rus.

19. Cocco M., Rice J.R. Pore pressure and poroelasticity effects in Coulomb stress analysis of earthquake interactions. JGR Solid Earth, 2002, vol. 107, no. B2, pp. ESE 2-1-ESE 2-17.

20. Mildon Z.K., Toda S., Walker J., Roberts G. Evaluating models of Coulomb stress transfer: Is variable fault geometry important? Geophysical Research Letters, 2016, vol. 43, no. 24, pp. 12,407-12,414.

21. Melnik E.A., Tataurova A.A., Olenchenko V.V., Glinskikh V.N. Studying of structural and material features of soils according to complex of seismic, gravity and geoelectric data. Natural and technical sciences, 2019, no. 2, pp. 66-70. In Rus.

Received: 12 March 2020.

\section{Information about the authors}

Antonina A. Tataurova, researcher, Institute of Petroleum Geology and Geophysics SB RAS.

Elena A. Melnik, Cand Sc., head of the laboratory, Institute of Petroleum Geology and Geophysics SB RAS.

Yurii P. Stefanov, Dr. Sc., leading researcher, Institute Petroleum Geology and Geophysics SB RAS. 